\title{
Temperature Induced Aggregation and Clouding in Humic Acid Solutions
}

\author{
Leah Shaffer and Ray von Wandruszka \\ Department of Chemistry, University of Idaho, Moscow, ID 83844-2343, USA \\ Correspondence should be addressed to Ray von Wandruszka; rvw@uidaho.edu
}

Received 8 December 2014; Accepted 30 January 2015

Academic Editor: Claire Richard

Copyright ( 2015 L. Shaffer and R. von Wandruszka. This is an open access article distributed under the Creative Commons Attribution License, which permits unrestricted use, distribution, and reproduction in any medium, provided the original work is properly cited.

\begin{abstract}
Humic acids in aqueous solution demonstrate inverse temperature-solubility relationships when solution conditions are manipulated to reduce coulombic repulsion among the humic polyanions. These effects were followed by dynamic light scattering (DLS) measurements of the resulting aggregates, as well as the addition of a polarity sensitive fluorescent probe (pyrene). The humic solutions could be primed for temperature induced clouding by carefully lowering the $\mathrm{pH}$ to a point where hydration effects became dominant. The exact value of the cloud point (CP) was a function of both $\mathrm{pH}$ and humate concentration. The CPs mostly lay in the range $50-90^{\circ} \mathrm{C}$, but DLS showed that temperature induced aggregation proceeded from approximately $30^{\circ} \mathrm{C}$ onward. Similar effects could be achieved by adding multivalent cations at concentrations below those which cause spontaneous precipitation. The declouding of clouded humate solutions could be affected by lowering the temperature combined with mechanical agitation to disentangle the humic polymers.
\end{abstract}

\section{Introduction}

Humic substances (HS) are the decay products of the total biota in the environment. They are grouped into three operationally defined categories: fulvic acid (FA), soluble at all pHs; humic acid (HA), soluble at $\mathrm{pH}>2$; and humin, insoluble at any $\mathrm{pH}$. HA and FA are environmentally the most important components, HA being the most abundant. They exist in both aquatic and terrestrial environments and interact extensively with other soil and water constituents. They are generally classified as natural polyanions with approximate molecular weight ranges of 500-2000 Da for FA and 2000-20,000 Da for HA. In view of these broad size ranges, a high degree of polydispersity exists in aqueous solutions of humic materials [1]. They are mildly acidic, primarily due to an abundance of carboxylic groups [2].

Dissolved HA will aggregate and precipitate when certain conditions are imposed.

(i) The $\mathrm{pH}$ is lowered below 2, causing the protonation of acidic groups and resulting in reduced coulombic repulsion between the humic species. (ii) Multivalent cations such as $\mathrm{Mg}^{2+}, \mathrm{Ca}^{2+}$, or $\mathrm{Sm}^{3+}$ that have the ability to form intra- and intermolecular ionic links between anionic (chiefly carboxyl) groups on the humates are added.

(iii) The ionic strength is raised sufficiently to result in a salting-out effect. Under these circumstances, the negative charges on the humic polyanions are shielded by the ionic content of the solution and coulombic repulsion among the humates is reduced. This leads to the formation of aggregates.

It has been observed previously [3] that aqueous HA solutions, not unlike those of nonionic surfactants, can undergo temperature induced clouding (TIC). This is a manifestation of an inverse temperature-solubility relationship, in which the heating of the solution to a temperature known as the cloud point $(\mathrm{CP})$ results in a cloudy appearance and eventually macroscopic phase separation. It is generally held that TIC in surfactant solutions is primarily due to the decrease of the relative permittivity, $\varepsilon_{r}$ (a.k.a. dielectric constant) of water with temperature, which leads to reduced hydration of the 
hydrophilic portions of the molecules and hence to their aggregation. The value of $\varepsilon_{r}$ for water at $20^{\circ} \mathrm{C}$ is 80.1 , while at $60^{\circ} \mathrm{C}$ it is 66.8 [4].

TIC is mostly encountered in nonionic surfactant solutions. Aqueous $\mathrm{HA}$, at circum-neutral $\mathrm{pH}$, is significantly ionic and does not display inverse solubility behavior. However, if one of the three conditions enumerated above is brought to bear-short of causing immediate precipitationthe solution conditions can be "tuned" to create a system that undergoes TIC. The likely cause of this is the partial suppression of the ionic character of HA, which makes effective hydration of the polar (but nonionic) portions of the molecules a more stringent prerequisite for solubility.

The work described in this communication focused on the details of TIC in aqueous solutions of humates, with emphasis on the solution conditions that promote the phenomenon. The bulk of the investigation was done with Latahco silt-loam humic acid (LSLHA), which has been shown to be an effective surfactant [5].

\section{Experimental Section}

2.1. Reagents and Solutions. Pyrene was obtained from Aldrich and purified by cold finger sublimation. Perylene was obtained from Arcos Organics. The humic materials used were both purchased commercially and isolated from a local soil. The former were obtained from the International Humic Substances Society (IHSS, 1991 Upper Buford Circle, Rm 439, St. Paul, USA) and included the following: Leonardite Humic Acid Standard (LHA), Soil Humic Acid Standard (SHA), Summit Hill Soil Humic Acid Reference (SHHA), and Minnesota Peat Fulvic Acid Reference (MPFA). Latahco silt-loam HA (LSLHA) was isolated in the laboratory from Latahco silt-loam (Argiaquic Xeric Argialbolls) according to the International Humic Substances Society (IHSS) procedure published on January 25, 1985. Humic solutions were prepared with doubly deionized water treated with a $0.22 \mu \mathrm{m}$ Millipore filter system. Dissolution was affected by the dropwise addition of a minimal amount of a $30 \%$ ammonium hydroxide solution. Reagent grade magnesium chloride hexahydrate was obtained from Baker, sodium chloride from Macron, samarium chloride from Aldrich, and hydrochloric acid from Fisher.

\subsection{Fluorescence Measurements. Fluorescence emission and} excitation measurements were obtained with a fluorolog3 fluorimeter manufactured by Horiba JobinYvon. Confirmation measurements were carried out with SLM Aminco 8100 and Hitachi U-4500 fluorescence spectrophotometers. For the determination of pyrene $I_{1} / I_{3}$ emission ratios, the solutions were excited at $240 \mathrm{~nm}$ and the emissions were measured at $372 \mathrm{~nm}\left(I_{1}\right)$ and $384 \mathrm{~nm}\left(I_{3}\right)$. An expandedrange thermometer purchased from Fisher Scientific was used to measure the temperature of the solution and confirm thermal equilibration before the fluorescent measurements were taken. Multiple spectra were taken to confirm results.

2.3. Particle Sizing. The size of humic aggregates was determined by dynamic light scattering measurements, using a
TABle 1: Cloud points $\left({ }^{\circ} \mathrm{C}\right)$ of LSLHA solutions at different $\mathrm{pH}$ values.

\begin{tabular}{lcccc}
\hline \multirow{2}{*}{$\mathrm{pH}$} & \multicolumn{4}{c}{ Concentration $(\mathrm{mg} / \mathrm{L})$} \\
& 40 & 60 & 80 & 100 \\
\hline 2.5 & 56 & 51 & 50 & 46 \\
3 & n.o. & 87 & 74 & 75 \\
3.5 & n.o. & n.o. & 95 & 90 \\
\hline
\end{tabular}

${ }^{*}$ Not observed.

Coulter N4 Plus Submicron Particle Sizer. The instrument employed a He-Ne laser light source $(632.8 \mathrm{~nm})$, and the scattering intensity was measured at a $90^{\circ}$ angle. Measurements were carried out in the unimodal mode [6]. Temperature control was implemented through an internal electronicPeltier heater/cooler with a precision of $\pm 0.2^{\circ} \mathrm{C}$ at $25^{\circ} \mathrm{C}$. Temperatures ranged from $20^{\circ}$ to $60^{\circ} \mathrm{C}$. Duplicate measurements of $300 \mathrm{~s}$ were taken to confirm results.

2.4. $\mathrm{pKa}$. The $\mathrm{pKa}$ of LSLHA was determined by titrating $125 \mathrm{~mL}$ of $40 \mathrm{mg} / \mathrm{L}$ LSLHA with a carbonate-free $0.04 \mathrm{M}$ $\mathrm{NaOH}$ solution, held at $22^{\circ} \mathrm{C}$ under nitrogen [7]. After dissolution of the HA sample with a minimal amount of base, the solution was acidified to $\mathrm{pH} 2.5$ with $\mathrm{HCl}$ prior to titration. The titration was performed with a Schott Titronic automated titrator, set to dispense $0.10 \mathrm{~mL}$ of titrant every $60 \mathrm{~s}$, and a Vernier glass combination electrode.

\section{Results and Discussion}

3.1. pH Adjustment. As noted in the Introduction, the occurrence of TIC in humic solutions is predicated on a reduction of the ionic character of the solute, allowing it to aggregate when the temperature is raised. In our first series of experiments, this was achieved by lowering the $\mathrm{pH}$ to 2.5 , which converted the humate to a form akin to a nonionic surfactant. At $20^{\circ} \mathrm{C}$, a $40 \mathrm{mg} / \mathrm{L}$ LSLHA solution did not cloud under these conditions, but when the temperature was increased, visible clouding occurred. The solution conditions under which the effect was observed are summarized in Table 1.

The data show that the cloud point decreased with increasing concentration and decreasing $\mathrm{pH}$, with a $\mathrm{pH}$ of 3.5 being an effective cut-off value above which no TIC occurred. In terms of the value of the $\mathrm{pK}_{1}$ of LSLHA, determined [7] to be 3.89 , lowering the $\mathrm{pH}$ to 2.5 for solubility reversal corresponds to the carboxyl groups of the material being approximately $96 \%$ protonated.

In view of the expectation that visible clouding would be preceded by progressive particle growth, the effect was quantitatively followed through fluorescent probe and dynamic light scattering measurements.

3.2. $I_{1} / I_{3}$ Ratio. Pyrene is a fluorophore that has been proven suitable as a probe for the study of aggregation in humic solutes [8]. The ratio of the first and third vibronic peaks in the pyrene emission spectrum $\left(I_{1} / I_{3}\right.$ ratio) is widely used to assess the polarity of the environment of the fluorophore [911]. When pyrene is sequestered by humic aggregates, in a manner analogous to micellization by synthetic surfactants, 


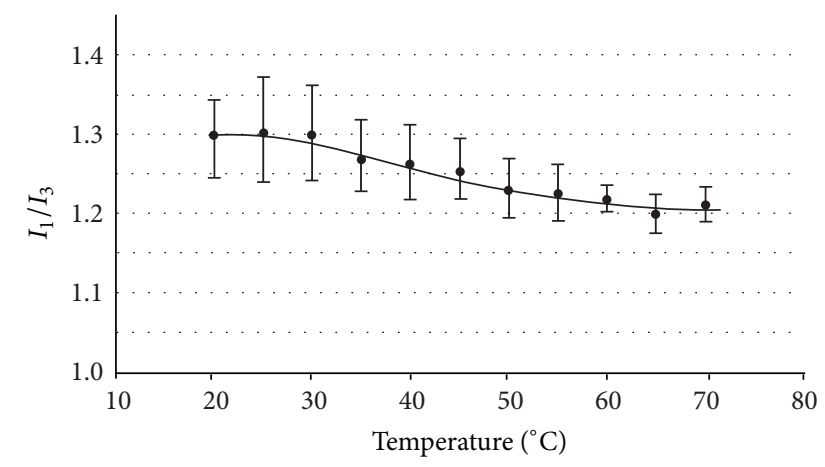

Figure 1: Variation of pyrene $I_{1} / I_{3}$ ratio with temperature in $40 \mathrm{mg} / \mathrm{L}$ LSLHA at $\mathrm{pH} 2.5$.

the $I_{1} / I_{3}$ ratio tends to decrease as a result of the reduced polarity experienced by the probe. In this manner, the ratio can be used to track the aggregation processes. Figure 1 shows the variation of the $I_{1} / I_{3}$ ratio with temperature leading up to the cloudpoint.

Two counteracting processes were in effect when the temperature of the primed humic solutions was increased: (i) thermal agitation (which tends to disrupt the aggregates) and (ii) dehydration (which promotes aggregate formation). The data in Figure 1 suggest that in the $20-35^{\circ} \mathrm{C}$ range the two effects largely canceled each other, resulting in a relatively flat curve. Beyond $35^{\circ} \mathrm{C}$, the $I_{1} / I_{3}$ ratio monotonically decreased, indicating that pyrene experienced an increasingly nonpolar environment. No such effect was observed with an aqueous pyrene solution in the absence of HA, so it can be concluded that temperature induced aggregation (TIA) of humate progressively took place as the solution was heated, culminating in TIC at $70^{\circ} \mathrm{C}$.

Direct evidence of TIA of dissolved humate was provided by dynamic light scattering measurements of an LSLHA solution at $\mathrm{pH}$ 2.5. The steady increase of average particle size with temperature shown in Figure 2 is consistent with $I_{1} / I_{3}$ ratio changes discussed above. It is important to note that the data in Figure 2 were obtained with a solution that did not contain pyrene, that is, a nonpolar solute that may act as a nucleation center for amphiphilic humates [2]. The particle growth observed under these circumstances lends further credence to the principal role of temperature in the aggregation process.

A further interesting observation was made when the particle sizes in a circumneutral solution of LSLHA containing pyrene were measured at a series of increasing temperatures.

Figure 3 shows that particle sizes in this solution, in which the humate was not significantly protonated and no temperature induced effects should be expected, decreased with temperature. This can be rationalized by considering that a similar solution without pyrene would have a particle size around $200 \mathrm{~nm}$, which is invariant with temperature. The presence of pyrene led to a small degree of hydrophobic association with humate, even in the absence of significant aggregation of the latter, giving rise to slightly increased particle sizes $(c a .275 \mathrm{~nm})$. Upon heating, however, these

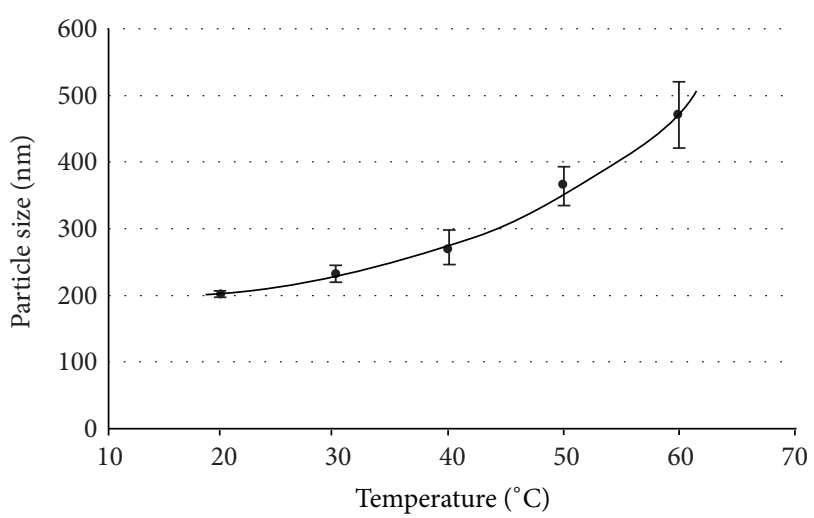

FIGURE 2: Variation of particle size with temperature in $40 \mathrm{mg} / \mathrm{L}$ LSLHA at $\mathrm{pH} 2.5$.

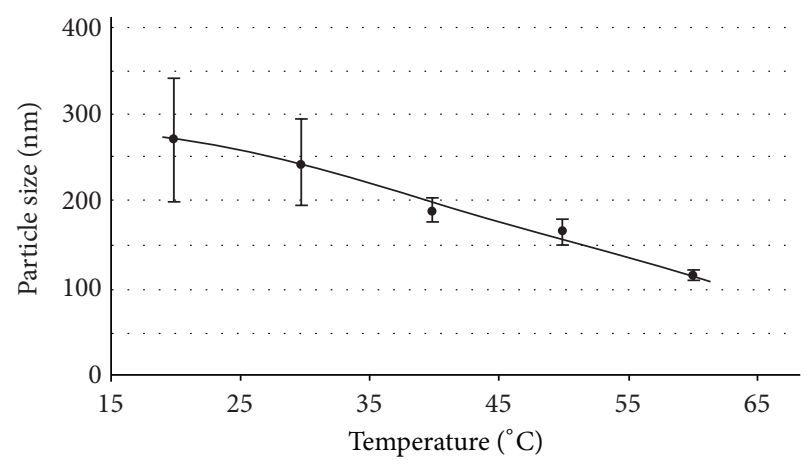

FIGURE 3: Variation of particle size of $40 \mathrm{mg} / \mathrm{L}$ LSLHA and $10^{-6} \mathrm{M}$ pyrene ( $\mathrm{pH} 7.5$ ).

weak aggregates were thermally disrupted, and the particle sizes decreased.

3.3. Effects of Cations. Raising the ionic strength of HA solutions can lead to aggregation and precipitation of the solutes. At ionic strengths below those that lead to complete salting out at room temperature, TIC/TIA effects can be observed. Monovalent cations are less effective than di- and trivalent ones, and in the case of $\mathrm{NaCl}$, TIC took place at $70^{\circ} \mathrm{C}$ in $60 \mathrm{mg} / \mathrm{L}$ LSLHA only when the salt was added in excess of $1 \mathrm{M}$. With a more modest $0.008 \mathrm{M} \mathrm{NaCl}$ concentration (twice that of the $\mathrm{MgCl}_{2}$ solutions, giving the same cationic strength) DLS particle sizing in a LSLHA solution showed no aggregation effects at temperatures up to $60^{\circ} \mathrm{C}$. In fact, as shown in Figure 4, a very slight decrease in particle size with temperatures was found. This size reduction, which was not observed in HA solutions without salt, can be attributed to the thermal disruption of electrolyte shielding [12] of the anionic charges on the humates. At low salt concentrations, this shielding process allowed for slight aggregation of the humic polymers [13], producing relatively small and fragile particles that disintegrated when thermally agitated.

In the presence of relatively low concentrations of $\mathrm{MgCl}_{2}$, the intra- and intermolecular crosslinking of humic entities by $\mathrm{Mg}^{2+}$ effectively primed the system for TIC. Table 2 shows 
TABLE 2: Cloud points $\left({ }^{\circ} \mathrm{C}\right)$ of humic materials in $0.004 \mathrm{M} \mathrm{MgCl}_{2}$ solutions.

\begin{tabular}{lcccc}
\hline HA & $40 \mathrm{mg} / \mathrm{L}$ & $60 \mathrm{mg} / \mathrm{L}$ & $80 \mathrm{mg} / \mathrm{L}$ & 51 \\
LSLHA & 54 & 50 & 55 & $100 \mathrm{mg} / \mathrm{L}$ \\
Leonardite HA (LHA) & 75 & 67 & 65 & 57 \\
Soil HA (SHA) & 69 & 65 & n.o. & 45 \\
Summit Hill HA (SHHA) & n.o. $^{*}$ & n.o. & n.o. & n.o. \\
Minnesota Peat FA (MPFA) & n.o. & n.o. & n.o. \\
\hline
\end{tabular}

${ }^{*}$ No cloud point observed.

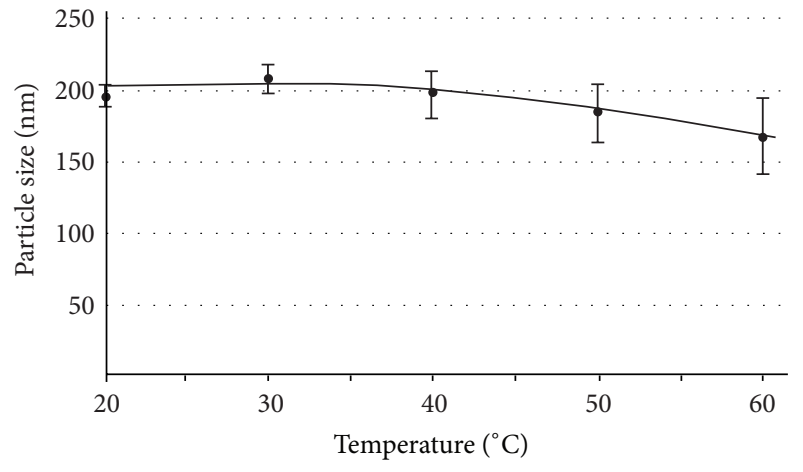

FIGURE 4: Variation of particle size with temperature of $40 \mathrm{mg} / \mathrm{L}$ LSLHA and $0.008 \mathrm{M} \mathrm{NaCl}$ (pH 7.5).

a summary of the cloud points of different concentrations of LSLHA in $0.004 \mathrm{M}$ aqueous $\mathrm{MgCl}_{2}$ solutions. For comparison, values of IHSS humic and fulvic acids are also shown.

It can be seen that LSLHA clouded at relatively low temperatures and that the cloud points were similar over the concentration range. More varied and higher cloud points were found with LHA and SHA, while, interestingly, SHHA and MPFA did not cloud at all. It is not immediately clear why the latter two could not be primed to behave as nonionic surfactants. The reason may lie in the fact that these are aquatic materials with a possibly less pronounced amphiphilic nature.

Figures 5 and 6 show the respective temperature responses of particle size and the pyrene $I_{1} / I_{3}$ ratio in LSLHA solutions containing $\mathrm{MgCl}_{2}$. Both the lowering of the $\mathrm{pH}$ and the addition of $\mathrm{Mg}^{2+}$ rendered the LSLHA solute sufficiently nonionic to undergo TIA/TIC. The CP was lower in the latter case, showing that the intra- and intermolecular crosslinking caused by divalent cation were more effective in readying the system for temperature induced effects. LSLHA has a relatively flexible backbone allowing the $\mathrm{Mg}^{2+}$ ions to "pull together" and crosslink dispersed portions of the humic polyanions, thereby forming a pseudomicellar particle [14].

The decrease of the pyrene $I_{1} / I_{3}$ ratio from 1.39 to 1.27 in the $20-40^{\circ} \mathrm{C}$ range (Figure 6 ) suggests that the probe became more sequestered in the humic environment in the presence of $\mathrm{Mg}^{2+}$ as the temperature was raised. The amount of aggregation beyond this temperature resulted in light scattering that interfered with the pyrene fluorescent measurements. However, the dynamic light scattering measurements indicated a change in particle size from $350 \mathrm{~nm}$ at $20^{\circ} \mathrm{C}$ to $4650 \mathrm{~nm}$ at $60^{\circ} \mathrm{C}$.

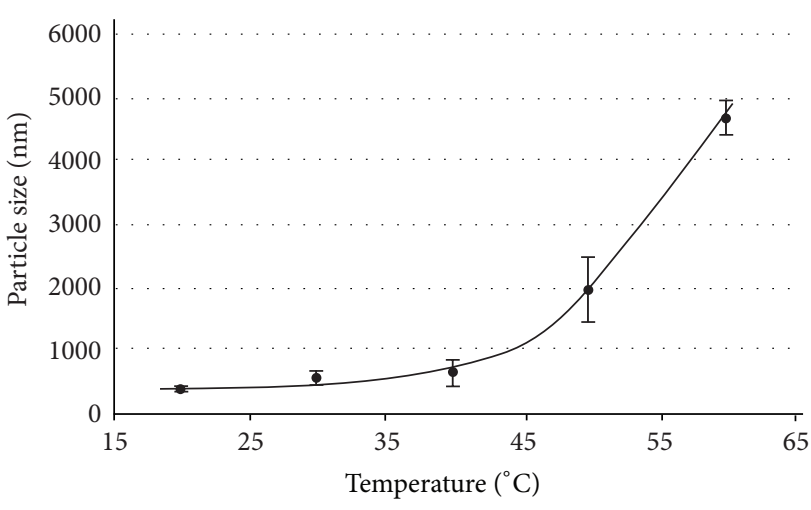

FIGURE 5: Variation of humate particle size with temperature in $40 \mathrm{mg} / \mathrm{L}$ LSLHA with $0.004 \mathrm{M} \mathrm{MgCl}_{2}$ (pH 7.5).

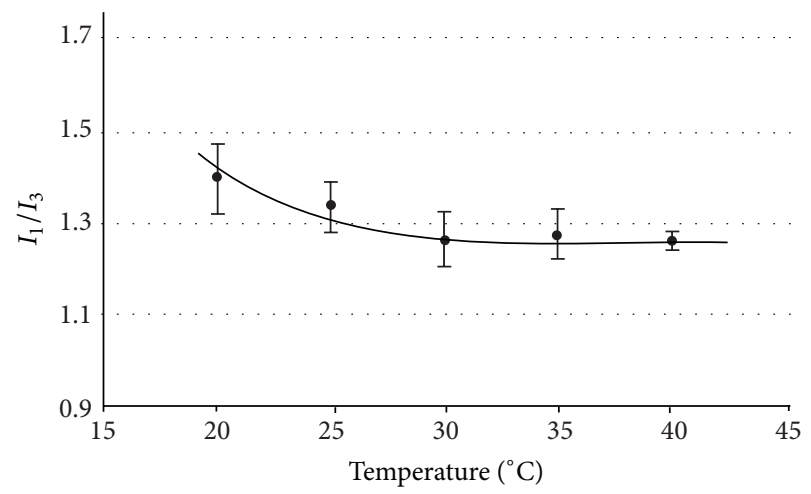

FIGURE 6: Variation of pyrene $I_{1} / I_{3}$ ratio with temperature in $40 \mathrm{mg} / \mathrm{L}$ LSLHA with $0.004 \mathrm{M} \mathrm{MgCl}_{2}$ (pH 7.5).

Priming HA solutions for TIA/TIC by adding $\mathrm{Mg}^{2+}$ ions likely involved intra- and intermolecular crosslinking of the humic polymers and required an excess of the metal ion. The acid content of LSLHA was determined by a conductimetric method [15] and found to be $6.52 \mathrm{meq} / \mathrm{g}$. When an equivalent amount of $\mathrm{Mg}^{2+}$ was added to LSLHA solutions, no TIC was observed at temperatures up to the boiling point. To assess the quantity of $\mathrm{Mg}^{2+}$ required to prime an aqueous LSLHA solution for TIC at $60^{\circ} \mathrm{C}, 3 \mu \mathrm{L}$ aliquots of a $1 \mathrm{M}$ $\mathrm{MgCl}_{2}$ solution were slowly added to $25 \mathrm{~mL}$ of a $40 \mathrm{mg} / \mathrm{L}$ LSLHA solution held at this temperature. It was found that it required an approximately 14 -fold excess of $\mathrm{Mg}^{2+}$ for visible clouding to occur. As noted above, an equivalent excess of positive charge provided by $\mathrm{Na}^{+}$ions did not lead to clouding, 


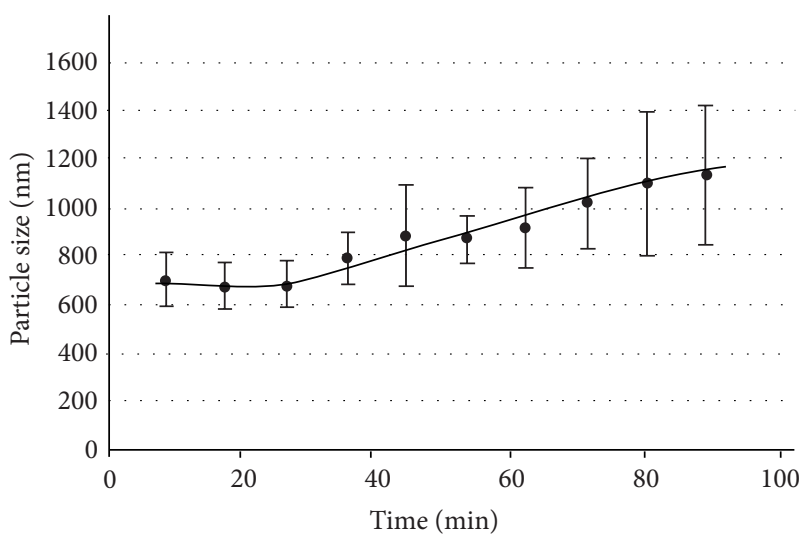

FIGURE 7: Time dependence of particle size in $40 \mathrm{mg} / \mathrm{L}$ LSLHA with $0.004 \mathrm{M} \mathrm{MgCl}_{2}$ at $30^{\circ} \mathrm{C}(\mathrm{pH} 7.5)$.

confirming that electrostatic shielding alone is not sufficient for TIC. Similar results were obtained with $\mathrm{CaCl}_{2}$. It appears that temperature induced effects caused by the addition of divalent cations were due to a combination of shielding and crosslinking.

3.4. Kinetic Effects. Aqueous humates in solutions of moderate ionic strength tend to precipitate when left at room temperature for extended periods. Figure 7 shows the development of particle size with time in a LSLHA/ $\mathrm{MgCl}_{2}$ solution at $30^{\circ} \mathrm{C}$. It can be seen that a gradual particle growth process took place; after about 90 min precipitation began. The progression of the coagulation was highly temperature dependent: at $40^{\circ} \mathrm{C}$ precipitation started after $26 \mathrm{~min}$; at $50^{\circ} \mathrm{C}$ it took $13 \mathrm{~min}$; and at $60^{\circ} \mathrm{C}$ it took $6.5 \mathrm{~min}$.

3.5. Declouding. When nonionic surfactants solutions are clouded by heating to the $\mathrm{CP}$, complete resolubilization can be affected by cooling: TIC is an easily reversible process with these solutes, allowing for effective rehydration and dissolution by reverting to lower temperatures. Declouding of the humic suspensions studied here did not proceed effortlessly. Redissolution could be achieved by lowering the temperature, but it also required vigorous agitation. To examine this behavior, two identical $60 \mathrm{mg} / \mathrm{L}$ LSLHA solutions containing $0.004 \mathrm{M} \mathrm{Mg}^{2+}$ were clouded by heating to $60^{\circ} \mathrm{C}$. One was kept at this temperature, and the other was cooled to $20^{\circ} \mathrm{C}$, and both were vigorously shaken on a wrist-action shaker. Solutions were allowed to cool to room temperature, which took 45-60 min. Under this regimen, the solution at $60^{\circ} \mathrm{C}$ remained clouded, while the turbidity of the $20^{\circ} \mathrm{C}$ solution dissipated. Similar behavior was observed with LSLHA solutions that were clouded by lowering the $\mathrm{pH}$ to 2.5 .

This declouding behavior can be rationalized by considering that temperature induced aggregation in humate solutions was initiated by dehydration, but the subsequent growth of the particles also involved entanglement and multiple interactions between the humic chains. At TIC, the particles responsible for the cloudy appearance were therefore held together by more than only hydrophobic interactions and could not be disrupted by merely increasing the dielectric constant of the aqueous solvent. Extensive agitation, together with a lower temperature, was needed to achieve redissolution.

\section{Conclusion}

The priming of humic solutions for temperature induced effects requires judicious reduction of charge on the humate polymers. With electrostatic repulsion diminished, warming the solution leads to dehydration that promotes the growth of humic aggregates. In the absence of other interactions it is likely that this could be reversed by simple cooling, but entanglement of the humic chains results in more durable particles that require mechanical disruption for complete redissolution.

\section{Conflict of Interests}

The authors declare that there is no conflict of interests regarding the publication of this paper.

\section{References}

[1] D. Šmejkalová and A. Piccolo, "Aggregation and disaggregation of humic supramolecular assemblies by NMR diffusion ordered spectroscopy (DOSY-NMR)," Environmental Science and Technology, vol. 42, no. 3, pp. 699-706, 2008.

[2] R. L. Wershaw, "Molecular aggregation of humic substances," Soil Science, vol. 164, no. 11, pp. 803-813, 1999.

[3] N. E. Palmer and R. von Wandruszka, "Dynamic light scattering measurements of particle size development in aqueous humic materials," Fresenius' Journal of Analytical Chemistry, vol. 371, no. 7, pp. 951-954, 2001.

[4] C. G. Malmberg and A. A. Maryott, "Dielectric constant of water from $0^{\circ}$ to $100^{\circ}$ C," Journal of Research of the National Bureau of Standards, vol. 56, no. 1, pp. 1-7, 1956.

[5] R. R. Engebretson and R. von Wandruszka, "Kinetic aspects of cation-enhanced aggregation in aqueous humic acids," Environmental Science \& Technology, vol. 32, no. 4, pp. 488-493, 1998.

[6] Product Literature, Coulter N4 Plus Sub micron Particle Sizer, Coulter Corporation, Miami, Fla, USA.

[7] J. D. Ritchie and E. M. Perdue, "Proton-binding study of standard and reference fulvic acids, humic acids, and natural organic matter," Geochimica et Cosmochimica Acta, vol. 67, no. 1, pp. 8596, 2003.

[8] C. Young and R. von Wandruszka, "A comparison of aggregation behavior in aqueous humic acids," Geochemical Transactions, vol. 2-5, pp. 16-20, 2001.

[9] D. Dong and M. Winnik, “The py scale of solvent polarities. Solvent effects on the vibronic fine structure of pyrene fluorescence and empirical correlations with $\mathrm{E}_{T}$ and $\mathrm{Y}$ values ," Photochemistry and Photobiology, vol. 35, no. 1, pp. 17-21, 1982.

[10] R. R. Engebretson and R. von Wandruszka, "The effect of molecular size on humic acid associations," Organic Geochemistry, vol. 26, no. 11-12, pp. 759-767, 1997.

[11] G. Basu Ray, I. Chakraborty, and S. P. Moulik, "Pyrene absorption can be a convenient method for probing critical micellar concentration ( $\mathrm{cmc}$ ) and indexing micellar polarity," Journal of Colloid and Interface Science, vol. 294, no. 1, pp. 248-254, 2006.

[12] R. A. Robinson and R. H. Stokes, Electrolyte Solutions, chapter 4, David \& Charles, Newton Abbot, UK, 2nd edition, 2002. 
[13] M. Drastík, F. Novák, and J. Kučerík, "Origin of heat-induced structural changes in dissolved organic matter," Chemosphere, vol. 90, no. 2, pp. 789-795, 2013.

[14] R. R. Engebretson and R. von Wandruszka, "Micro-organization in dissolved humic acids," Environmental Science and Technology, vol. 28, no. 11, pp. 1934-1941, 1994.

[15] J. Riggle and R. von Wandruszka, "Conductometric characterization of dissolved humic materials," Talanta, vol. 57, no. 3, pp. 519-526, 2002. 

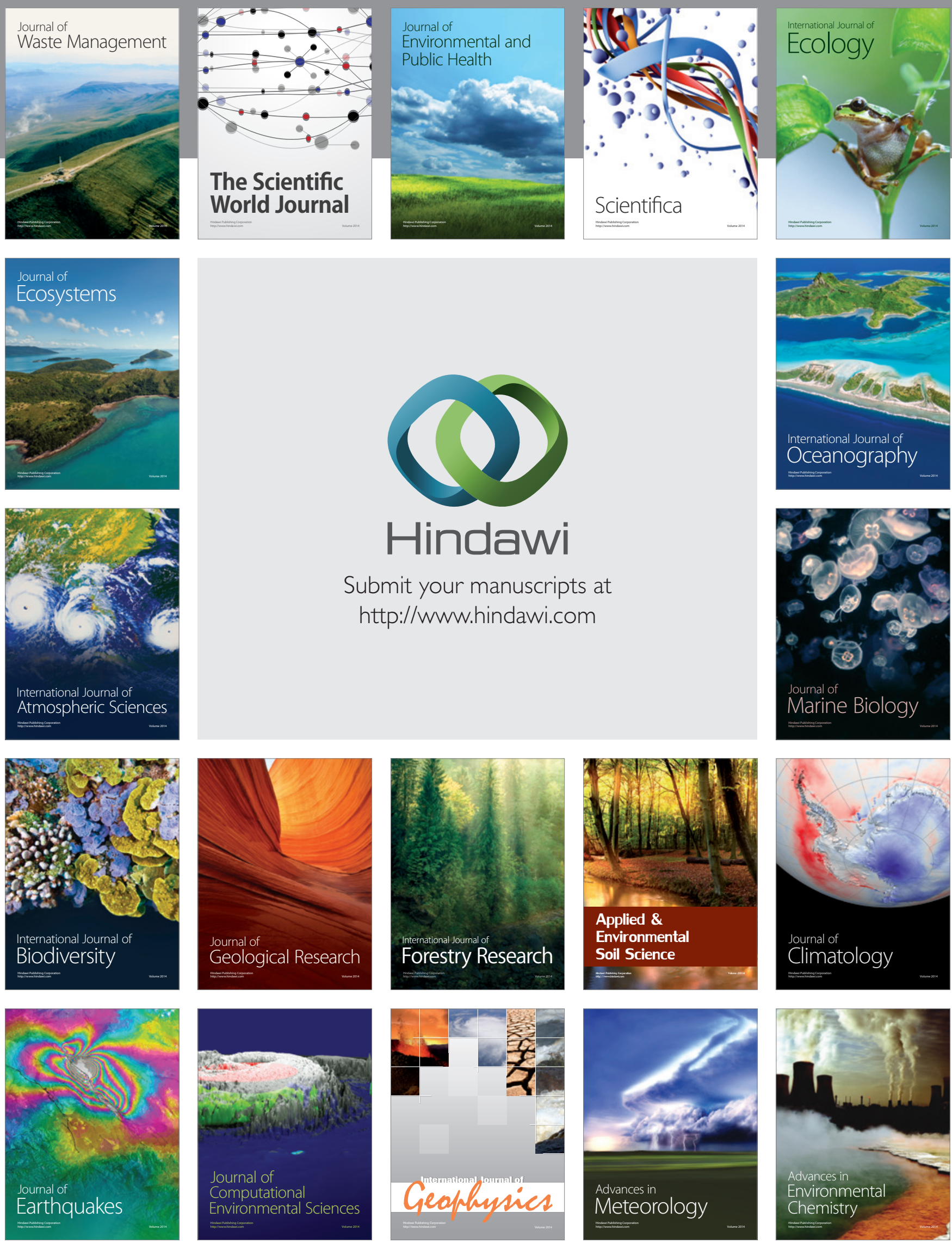